\title{
L'expérience au Yukon avec la COVID-19 : restrictions de voyage, variants et propagation entre les non-vaccinés
}

\author{
Sara McPhee-Knowles ${ }^{1 *}$, Bryn Hoffman², Lisa Kanary
}

\section{Résumé}

L'expérience du Yukon concernant la maladie à coronavirus 2019 (COVID-19) a été intéressante; le territoire a mis en place avec succès des restrictions de voyage pour limiter l'importation du virus et a déployé les vaccins rapidement par rapport à la plupart des administrations canadiennes. Cependant, la première vague de COVID-19 du Yukon en juin et juillet 2021 a fait déborder le système de santé en raison de la transmission généralisée parmi les enfants non-vaccinés, les jeunes et les adultes, malgré une forte participation à la vaccination et le port du masque obligatoire. Cette expérience met en lumière l'importance du soutien continu aux programmes de vaccination publique, de l'adoption généralisée de vaccins dans les populations pédiatriques, et l'assouplissement judicieux des interventions non pharmaceutiques dans toutes les administrations canadiennes à mesure qu'elles rouvrent alors que des variants plus contagieux apparaissent.
Cette oeuvre est mise à la disposition selon les termes de la licence internationale Creative Commons Attribution 4.0

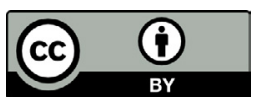

Affiliations

${ }^{1}$ Université du Yukon, Whitehorse, YK

2 École de médecine Queen's, Université Queen's, Kingston, ON

*Correspondance :

smcpheeknowles@yukonu.ca

Citation proposée : McPhee-Knowles S, Hoffman B, Kanary L. L'expérience au Yukon avec la COVID-19 : restrictions de voyage, variants et propagation entre les non-vaccinés. Relevé des maladies transmissibles au Canada 2022;48(1):19-23. https://doi.org/10.14745/ccdr.v48i01a03f

Mots-clés : COVID-19, territoire du Yukon, réponse à la pandémie, restrictions de déplacement, vaccination, éclosion, Nord du Canada

\section{Introduction}

La trajectoire de la maladie à coronavirus 2019 (COVID-19) dans I'ensemble du Canada a été inégale, et le territoire du Yukon, au Canada, est un cas intéressant. Du 22 mars 2020 au $1^{\text {er }}$ juin 2021, il y a eu 62 cas et deux décès attribuables au COVID-19 au Yukon (1). Une caractéristique importante de la stratégie de santé publique du Yukon était la mise en œuvre de restrictions de voyage, où, comme les provinces de l'Atlantique, les voyageurs qui entrent dans le territoire devaient s'isoler pendant 14 jours. Le Yukon comporte une population petite et dispersée et, grâce à peu de points d'accès, a pu imposer des restrictions de voyage pour limiter les cas importés. Toutefois, en juin et juillet 2021, peu après la levée de certaines restrictions, le Yukon a connu sa première vague de COVID-19 avec transmission communautaire, malgré le taux de vaccination le plus élevé au Canada à la fin du

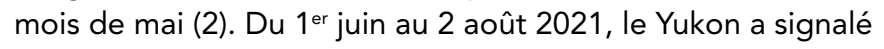
541 nouveaux cas de COVID-19 dans le cadre de trois éclosions distinctes et six décès dans une population d'environ 42000 (3). La plupart des personnes qui sont tombées malades n'étaient pas vaccinées (4), avec seulement $14 \%$ des cas entièrement vaccinés, et aucun des patients atteints de COVID-19 qui sont décédés n'a été entièrement vacciné (1). Le 28 juillet 2020, le gouvernement a rapporté qu'un total de 52 personnes ont été hospitalisées pendant cette vague; de ce groupe, 43 n'ont pas été vaccinés ou seulement partiellement vaccinés. Quatorze cas, dont 11 non-vaccinés, étaient dans un état critique et ont été évacués pour urgence médicale vers des centres plus vastes (5). Dans le présent commentaire, nous présentons l'expérience au Yukon avec la pandémie de COVID-19 et soulignons les leçons tirées de sa dernière vague de COVID-19 en juin et juillet 2021.

\section{Contexte}

Le Yukon et le deuxième territoire de compétence le plus petit du Canada par population. Environ $75 \%$ de la population vit à Whitehorse, la capitale du territoire, et le reste, dans 15 petites collectivités (3). Il y a trois hôpitaux. Whitehorse General, le plus grand, compte 56 lits, une gamme de services incluant une unité de soins intensifs à quatre lits, et accueille 32000 visites aux urgences et 3703 admissions par année. Deux hôpitaux communautaires à Watson Lake et Dawson City ont des services d'urgence et six unités de malades admis de six lits avec 112 et 80 admissions par année et 2627 et 2812 visites aux urgences par an, respectivement (6). Cette capacité d'hospitalisation globale est historiquement adéquate pour la population; toutefois, l'évacuation médicale ou les déplacements pour raison 
médicale sont souvent requis pour les cas de grande acuité ou pour les cas nécessitant des soins spécialisés (7). Cela fait que le Yukon est plus à risque pendant la pandémie de COVID-19, car une éclosion importante pourrait dépasser la capacité des services de santé. Si des cas surviennent également dans d'autres territoires de compétence, il pourrait être impossible d'évacuer les patients à des centres plus grands, comme Vancouver ou Edmonton (8).

À la suite de la décentralisation en 2003, le gouvernement territorial du Yukon a assumé la responsabilité de la santé publique, de concert avec d'autres pouvoirs provinciaux, du gouvernement fédéral canadien. Onze des 14 Premières Nations du Yukon sont autonomes et peuvent s'acquitter de leurs responsabilités du gouvernement territorial, y compris certaines liées à la santé, après avoir adopté leur propre législation (9). Dans le recensement de 2016, environ 23 \% de la population a indiqué avoir une ascendance autochtone (10). L'économie du Yukon est largement fondée sur le gouvernement; le Yukon dépend fortement des transferts fédéraux (11). L'exploitation minière, les services et le tourisme sont également des moteurs importants. En raison des exigences d'auto-isolement après le voyage, le tourisme a diminué de $25 \%$ au premier trimestre de 2020 par rapport à 2019 (12). Le contexte démographique, économique et institutionnel unique du Yukon a influencé l'intervention en cas de pandémie.

\section{Réponse à la pandémie}

Le gouvernement du Yukon, grâce à son statut de " protoprovince » (13), a dirigé l'intervention en cas de pandémie; le Conseil des Premières Nations du Yukon, qui représente les gouvernements des Premières Nations du Yukon, a également joué un rôle dans la coordination et la communication. Très tôt le gouvernement du Yukon a adopté des restrictions typiques en matière de santé publique, comme la restriction des rassemblements, la fermeture des bars et des services de soins personnels, et la suspension des services de soins de santé. Les premiers cas de COVID-19 au Yukon ont été annoncés le 22 mars 2020 (figure 1), après la mise en place des restrictions. Restriction des déplacements à l'extérieur du territoire au 22 mars 2020, nombre limité de cas d'importation au Yukon : un isolement de 14 jours était requis pour tous les voyageurs qui entraient dans le territoire. On a demandé aux Yukonnais de limiter leurs déplacements dans les collectivités rurales, et certains gouvernements des Premières Nations ont établi des points de contrôle dans leurs territoires traditionnels. Un arrêté ministériel visant à renforcer les contrôles aux frontières a été publié le 2 avril 2020 pour faire respecter les exigences d'isolement aux points d'entrée frontaliers (14). Une "bulle» de voyage avec la Colombie-Britannique a été établie le

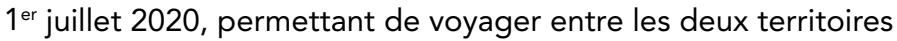
sans isolement; toutefois, la bulle a pris fin le 20 novembre 2020, après que les cas aient commencé à augmenter au Yukon (15). Ces restrictions de voyage ont effectivement empêché une éclosion majeure de COVID-19 au Yukon pendant la première année de la pandémie. Le port obligatoire du masque pour les Yukonnais de plus de cinq ans dans les lieux publics a été décrété le $1^{\text {er }}$ décembre 2020 - l'une des dernières administrations canadiennes à imposer le port du masque (16) depuis que le Yukon avait connu un nombre aussi limité de cas de COVID-19 (figure 1).

Les premières doses du vaccin de Moderna contre la COVID-19 ont été administrées au Yukon le 4 janvier 2021, plus tôt que les provinces canadiennes en raison de la capacité hospitalière limitée du territoire. Des équipes de vaccination mobile ont été déployées dans des collectivités à l'extérieur de Whitehorse (17). En date du 22 mai 2021, 55,22 \% de la population totale était entièrement vaccinée (2). Les exigences d'auto-isolement ont été levées le 25 mai 2021 pour les voyageurs internes

Figure 1 : Calendrier des événements clés liés à la pandémie de COVID-19 au Yukon, Canada, du $1{ }^{\text {er }}$ mars 2020 au 31 juillet 2021

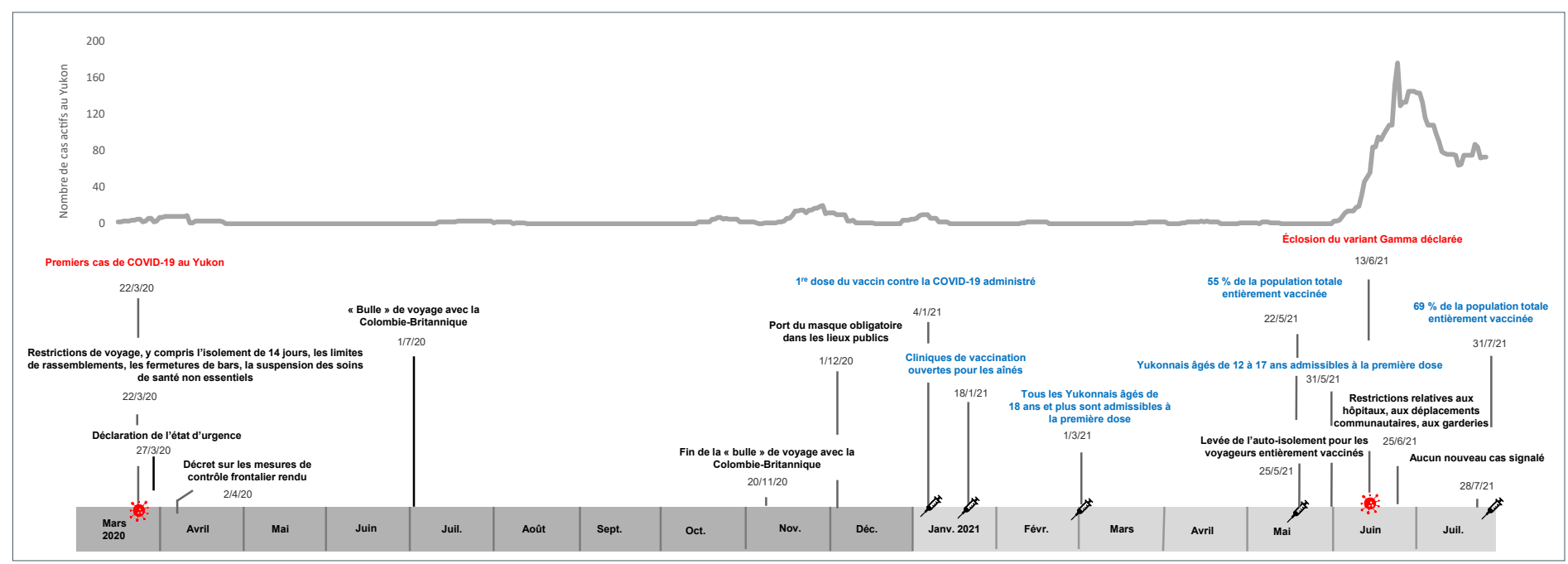

Abréviation : COVID-19, maladie à coronavirus 2019 
entièrement vaccinés ou pour les Yukonnais entièrement vaccinés qui retournent après un voyage intérieur (figure 1) (18). Cette annonce a créé une incitation supplémentaire à la vaccination.

Peu après la levée des exigences d'auto-isolement pour les voyageurs, une éclosion du variant Gamma a été déclarée le 13 juin 2021 (figure 1) (19). La transmission s'est produite lors des célébrations de fin d'études secondaires, dans les bars (20), les garderies et au refuge d'urgence de Whitehorse; la transmission se faisait principalement chez des adultes, des jeunes et des enfants non vaccinés (4). La saison de remise de diplôme a facilité la transmission de la maladie parce que les diplômés et les membres de la famille ont voyagé entre les collectivités et ont assisté à des célébrations informelles, non masquées et à des réunions plus vastes et organisées avec les mesures de COVID-19 en place. En fait, l'éclosion peut être liée à une seule personne infectée qui a participé à une grande fête (20). Le 15 juillet 2021, 240 des 414 cas ont été confirmés comme étant le variant Gamma (21).

Le médecin hygiéniste en chef du Yukon a encouragé les personnes qui ne sont pas encore vaccinées à prendre des rendez-vous et tous les Yukonnais à " rester à six " personnes pour des rassemblements, mais a abaissé les limites de rassemblement officielles à 10 à l'intérieur avec des masques et 20 à l'extérieur (22). Parmi les autres mesures de santé publique, mentionnons l'annulation de certains événements de remise de diplômes (23), encourager fortement les parents à garder les enfants à la maison loin des garderies (22) et l'augmentation des restrictions par la Régie des hôpitaux du Yukon (4). Des cas étaient présents dans la plupart des collectivités du Yukon. De nombreuses Premières Nations du Yukon ont demandé aux voyageurs de s'abstenir de voyager. Les équipes de recherche des contacts, de dépistage et de vaccination étaient à leur capacité; le premier ministre a demandé un soutien supplémentaire du gouvernement fédéral (22). Le 28 juillet 2021, aucun nouveau cas n'a été signalé pour la première fois depuis le 5 juin 2021; au 31 juillet 2021, 68,63 \% de la population totale était entièrement vaccinée (figure 1) (2). Toutes les autres restrictions en matière de santé publique ont été levées le 4 août 2021, y compris l'exigence pour les voyageurs qui n'ont pas été vaccinés de s'isoler et de porter obligatoirement un masque à l'intérieur (24); cette décision a fait l'objet de critiques publiques, notamment de la part du chef de la Première Nation Kwanlin Dün (25).

\section{Leçons apprises}

On peut tirer certains enseignements de l'expérience du Yukon avec la COVID-19. La première est l'importance d'atténuer les importations de cas au moyen d'exigences d'auto-isolement pour les voyageurs qui entrent dans une région. À titre d'exemple, des études de modélisation pour Terre-Neuve ont démontré que sans l'introduction d'une exigence d'auto-isolement pour les voyageurs, il y aurait eu 12,4 fois plus de cas de COVID-19 au cours des premières semaines de la pandémie (26). La gestion des importations de cas est essentielle dans les petites administrations ayant une capacité d'hospitalisation limitée, comme le Yukon, les Territoires du Nord-Ouest et le Nunavut. Les régions éloignées peuvent également être en mesure de surveiller plus facilement les points d'entrée que les grands centres mieux connectés. Ce choix de politique signifie que l'industrie du tourisme a été touchée de façon disproportionnée par rapport à de nombreuses autres entreprises du Yukon, et elle ne sera pas durable à long terme dans ces conditions.

Une autre considération pour le Yukon, et d'autres territoires de compétence qui n'ont pas connu un grand nombre de cas de COVID-19 plus tôt dans la pandémie, était que l'adoption de plans de réouverture signifiait une augmentation des cas et donc des risques, comparativement aux secteurs qui ont rouvert en raison de la diminution du nombre de cas. Il y avait aussi la question du moment de la réouverture, qui a eu lieu peu de temps avant la cérémonie d'obtention des diplômes, alors qu'il y avait une augmentation des déplacements entre les collectivités du Yukon, a probablement contribué à la propagation rapide de l'épidémie.

Un point de vue central, d'importance à la fin de 2021, alors que d'autres administrations canadiennes rouvraient leurs portes, est que les taux élevés de vaccination et le port du masque obligatoire n'étaient pas suffisants pour prévenir les éclosions dans les populations non vaccinées, ce qui exerce une pression sur le système de santé local. Les étudiants du secondaire, qui font partie du groupe d'âge des 12 à 17 ans, n'étaient pas admissibles à la vaccination avant la réouverture (27), tandis que les enfants en garderie étaient trop jeunes pour être vaccinés, et certaines communautés avaient des taux de vaccinations plus faibles que d'autres (28). Les décisions politiques fondées sur un pourcentage global de personnes vaccinées ignorent que les groupes non vaccinés, en raison de leur âge ou de leur mode de vie, ont tendance à interagir, ce qui facilite la propagation de la maladie.

Bien que les enfants ne souffrent généralement pas de conséquences graves secondaire à la COVID-19 (29), les garderies étaient des points chauds durant l'éclosion estivale au Yukon (22). Pour gérer l'éclosion, le médecin hygiéniste en chef a recommandé que les parents qui ne sont pas des travailleurs essentiels gardent leurs enfants à la maison si possible. Étant donné que les parents ont une capacité limitée de travailler sans avoir accès à des services de garde d'enfants fiables et que les femmes assument généralement une plus grande part du fardeau des responsabilités en matière de garde d'enfants, ce qui a été exacerbé par la pandémie (30), cette recommandation a eu des conséquences économiques qui ont eu des répercussions disproportionnées sur les femmes. Actuellement, les enfants de moins de cinq ans ne sont pas admissibles à la vaccination et sont donc vulnérables aux variantes plus contagieuses du COVID-19. 


\section{Conclusion}

On peut tirer une leçon de l'expérience du Yukon : les restrictions de déplacement dans la région éloignée ont permis d'atténuer efficacement l'importation de la maladie au début de la pandémie, mais une fois ces restrictions éliminées, le variant Gamma hautement contagieux a circulé dans les populations non vaccinées. Même avec un taux de vaccination élevé et le port du masque, des éclosions se sont produites qui ont surchargé la capacité de la santé publique et des soins de santé. Il s'agit d'une mise en garde pour d'autres administrations, car les mesures de santé publique sont éliminées et l'adoption des vaccins a plafonné. Le fardeau prolongé de la maladie COVID-19 au Yukon pourrait inclure des conséquences imprévues, comme un plus grand nombre de cas pédiatriques, la fermeture des garderies et des écoles et leurs répercussions connexes sur la santé économique et mentale, plus de difficultés pour les entreprises, et un système de santé surchargé. Ces répercussions sont également observées ailleurs au Canada au cours de la quatrième vague. Une vaccination généralisée dans tous les groupes d'âge et les communautés est nécessaire pour réduire la gravité des futures vagues de COVID-19.

\section{Déclaration des auteurs}

Tous les auteurs ont contribué à la conceptualisation, à la rédaction, ainsi qu'à l'examen et la révision de ce commentaire.

Le contenu et les points de vue exprimés dans cet article sont ceux des auteurs et ne reflètent pas nécessairement ceux du gouvernement du Canada.

\section{Intérêts concurrents}

Aucun.

\section{Remerciements}

Les auteurs tiennent à remercier R. Hulstein pour ses commentaires réfléchis sur un avant-projet.

\section{Financement}

Le soutien financier pour cette étude a été fourni par la subvention pour des activités universitaires de l'Université du Yukon.

\section{Références}

1. Gouvernement du Yukon. Le 2 août 2021 : le point sur les cas de COVID-19. Whitehorse (YT): Gouvernement du Yukon; 2021 (accédé 2021-08-03). https://yukon.ca/fr/news/ le-2-aout-2021-le-point-sur-les-cas-de-covid-19

2. Agence de la santé publique du Canada. Vaccination contre la COVID-19 au Canada. Ottawa (ON) : ASPC; 2021 (accédé 2021-08-11). https://sante-infobase.canada.ca/covid-19/ couverture-vaccinale/

3. Yukon Bureau of Statistics. Population Report, Third Quarter 2020. Whitehorse (YT): Government du Yukon; 2020. https://yukon.ca/sites/yukon.ca/files/ybs/ populationq3_2020_r_0_0.pdf

4. Gouvernement du Yukon. Le 27 juin 2021 : nouveaux cas de COVID-19 confirmés et nouvelles directives concernant I'éclosion. Whitehorse (YT) : Gouvernement du Yukon; 2021 (accédé 2021-08-11). https://yukon.ca/fr/news/june-27-2021. new-covid-19-cases-confirmed-further-guidance-outbreakprovided

5. Government of Yukon. COVID-19 Facebook live update: July 28, 2021. Whitehorse (YT): Government of Yukon; 2021 (accédé 2021-11-13). https://yukon.ca/sites/yukon.ca/files/ eco/eco-live-update-transcript-july-28-2021.pdf

6. La Régie des hôpitaux du Yukon. Nos hôpitaux. Yukon : RHY; 2021 (accédé 2021-04-29). https://yukonhospitals.ca/fr/ yukon-hospital-corporation/our-hospitals

7. Young TK, Tabish T, Young SK, Healey G. Patient transportation in Canada's northern territories: Patterns, costs and providers' perspectives. Rural Remote Health 2019;19(2):5113. DOI PubMed

8. Cunningham VL. The evolution of the Yukon Medevac Program in an environment of fiscal restraint. CMAJ 1999;161(12):1559-62. PubMed

9. Dacks G. Implementing First Nations Self-Government in Yukon: Lessons for Canada. Can J Polit Sci 2004;37(3):671-94. DOl

10. Anora A. Le Yukon : Beau, complexe et en évolution. Ottawa (ON) : StatCan (modifié 2021-03). https://www150.statcan. gc.ca/n1/pub/11-631-x/11-631-x2018006-fra.htm

11. Gouvernement du Canada. Principaux transferts fédéraux. Ottawa (ON) : Gouvernement du Canada; 2014 (accédé 2021-05-01). https://www.canada.ca/fr/ministere-finances/ programmes/transferts-federaux/principaux-transfertsfederaux.html

12. Gouvernement du Yukon. Données et rapports sur le tourisme et les visiteurs. Whitehorse (YT) : Gouvernement du Yukon; 2019 (accédé 2021-04-29). http://yukon.ca/fr/ tourism-statistics\#retombees-economiques 
13. Cameron K. Yukon, the 'proto-province': Thoughts on an interesting constitutional parallel. Ottawa (ON): Northern Public Affairs (modifié 2016-05-31; accédé 2018-11-08). http://www.northernpublicaffairs.ca/index/yukon-the-protoprovince-thoughts-on-an-interesting-constitutional-parallel/

14. Government of Yukon. A Path Forward: Yukon's Plan for Lifting COVID-19 Restrictions. Whitehorse (YT): Government of Yukon; Mar 2021. https://yukon.ca/sites/yukon.ca/ files/eco/eco-path-forward-yukon-plan-lifting-covid-19restrictions-march-5-2021.pdf

15. d'Entremont D, Howells L. Tears and "divorce": Yukon bursts B.C. travel bubble amid rising COVID-19 cases. CBC News. Nov 19, 2020 (accédé 2021-07-30). https://www. cbc.ca/news/canada/north/yukon-officials-update-isolationrestrictions-tighten-1.5808058

16. Gouvernement du Yukon. COVID-19 et port du masque. Whitehorse (YT) : Gouvernement du Yukon; 2020 (accédé 2021-07-30). https://yukon.ca/fr/sante-et-bien-etre/votresante-covid-19/port-du-masque-au-yukon

17. d'Entremont D. Here's when and where Yukoners can get vaccinated against COVID-19. CBC News. 2021 (accédé 2021-08-14). https://www.cbc.ca/news/canada/north/yukonofficials-covid-19-update-1.5864875

18. Gouvernement du Yukon. Levée des restrictions de santé publique à partir du 25 mai. Whitehorse (YT) : Gouvernement du Yukon (modifié 2021-05-21; accédé 2021-08-11). https://yukon.ca/fr/news/public-healthrestrictions-be-lifted-may-25

19. Gouvernement du Yukon. La médecin hygiéniste en chef par intérim déclare une éclosion de COVID-19. Whitehorse (YT) : Gouvernement du Yukon; 2021 (accédé 2021-07-31). https:// yukon.ca/fr/news/la-medecin-hygieniste-en-chef-par-interimdeclare-une-eclosion-de-covid-19

20. CBC News. Yukon COVID-19 outbreak linked to single party. CBC; 2021 (accédé 2021-08-11). https://www.cbc.ca/player/ play/1913004611747

21. Government of Yukon. COVID-19 Facebook live update: July 14, 2021 (accédé 2021-11-12). https://yukon.ca/sites/ yukon.ca/files/eco-live-update-transcript-july-14-2021.pdf
22. Government of Yukon. June 30, 2021 COVID-update. YouTube: June 30, 2021 (accédé 2021-08-04). https://www.youtube.com/watch?v=AF99_rcaS2w\&list=PL4 B1jekdBO2rkKxe2fdbOZFlocNRpWZAH\&index=95

23. Gouvernement du Yukon. 11 juin 2021 : Le bal de finissants de l'École secondaire de Porter Creek est annulé. Whitehorse (YT) : Gouvernement du Yukon; 2021 (accédé 2021-08-11). https://yukon.ca/fr/news/11-juin-2021-le-balde-finissants-de-lecole-secondaire-de-porter-creek-estannule

24. Gouvernement du Yukon. Levée de restrictions sanitaires au vu de la hausse des taux de vaccination. Whitehorse (YT) : Gouvernement du Yukon; 2021 (accédé 2021-07-30). https://yukon.ca/fr/news/additional-covid-19-restrictions-belifted-vaccine-rate-increases

25. CBC News. Kwanlin Dün pushes back on Yukon reopening plan. CBC; 2021 (accédé 2021-08-11). https://www.cbc.ca/ news/canada/north/kwanlin-d\%C3\%BCn-reopening-toosoon-1.6114522

26. Hurford A, Rahman P, Loredo-Osti JC. Modelling the impact of travel restrictions on COVID-19 cases in Newfoundland and Labrador. R Soc Open Sci 2021;8(6):202266. DOl PubMed

27. Gouvernement du Yukon. Début de la vaccination contre la COVID-19 pour les jeunes le 31 mai. Whitehorse (YT) : Gouvernement du Yukon; 2021 (accédé 2021-08-12). https:// yukon.ca/fr/news/covid-19-vaccination-clinics-youth-beginmay-31

28. Gouvernement du Yukon. Relevons la manche : I'avenir s'annonce radieux. Whitehorse (YT) : Gouvernement du Yukon; 2021 (accédé 2021-04-30). https://yukon.ca/fr/voilanotre-chance

29. Cui X, Zhao Z, Zhang T, Guo W, Guo W, Zheng J, Zhang J, Dong C, Na R, Zheng L, Li W, Liu Z, Ma J, Wang J, $\mathrm{He}$ S, Xu Y, Si P, Shen Y, Cai C. A systematic review and meta-analysis of children with coronavirus disease 2019 (COVID-19). J Med Virol 2021;93(2):1057-69. DOI PubMed

30. Power K. The COVID-19 pandemic has increased the care burden of women and families. Sustain Sci Pract Policy. 2020;16(1):67-73. DOI 